\title{
Sensibilité aux Pythium et mauvaise germination en sol froid chez le haricot (Phaseolus vulgaris $L$ )
}

\author{
JP Ginoux 1, CM Messiaen 2* \\ ${ }^{1}$ ASL Agroparc BP 119, F 84144 Montfavet Cedex; \\ 2 INRA, unité de recherche et formation en biologie et pathologie végétales, ENSA-INRA, \\ place Viala, F 34060 Montpellier Cedex, France
}

(Reçu le $1^{\text {er }}$ octobre 1992; accepté le 5 janvier 1993)

\begin{abstract}
Résumé - Les manques à la levée observés sur haricot en semis précoce au printemps sont principalement dus aux attaques de Pythium. D'importantes différences de sensibilité peuvent être mises en évidence entre variétés par des méthodes de contamination les mettant en présence de souches de Pythium douées de divers niveaux d'agressivité. Les variétés à grain coloré sont, de façon générale, moins sensibles que celles à tégument incolore, et parmi celles-ci les flageolets verts sont les plus sensibles. L'étude de la descendance d'un croisement (noir résistant x flageolet vert) met en évidence l'effet sensibilisant de 2 gènes récessifs, l'un conditionnant l'absence de coloration, l'autre le caractère "flageolet". Cependant, il y a aussi des différences de sensibilité entre variétés à grains noirs. Les variétés plus sensibles exsudent plus de sucres et de composés azotés en cours de germination, leurs téguments sont moins riches en leuco-anthocyanes.

L'aptitude physiologique à germer et à croître à basse température intervient également dans la réussite des semis précoces. Elle a fait, elle aussi, l'objet d'une étude variétale qui met en évidence les qualités de la variété Vernandon.
\end{abstract}

haricot $=$ Phaseolus vulgaris $/$ germination $/$ sol froid $/$ Pythium

Summary - Pythium susceptibility and germination failure in common beans (Phaseolus vulgaris) sown in cold soils. Bean growers and the canning industry would appreciate the improved success of early sowings of common beans, which are frequently damaged by Pythium spp. Twenty strains were collected and classified for agressivity and taxonomy: $P$ ultimum (most of them very agressive), $P$ sylvaticum (most of them fairly agressive), and palmatecoralloid strains, which are weakly agressive. Laboratory methods are described for inoculation of bean seeds with Pythium, analysis of bean seed exudates and estimation of leucoanthocyanin content of the seed coat.

Significant differences in Pythium susceptibility could be demonstrated between bean cultivars. Black beans, and also some red, buff-colored and mottled beans were more resistant than white ones, amongst which differences in susceptibility could be demonstrated by the use of weakly aggressive Pythium strains. The highest susceptibility occured for the French flageolet vert cultivars. A study of the progeny of a cross between PI226895 (the most resistant black bean) and Elsa (a 'flageolet vert') demonstrate the increases in susceptibility caused by the 2 recessive genes inducing the absence of coloration and flageolet quality. High levels of resistance could be found in buff-colored as well as in black-seeded lines in the progeny of this cross. At the biochemical level susceptibility is associated with high level of soluble substances (sugars, nitrogen compounds) in seed exudates during imbibition, low content in leucoanthocyanins, and seed coat thinness. The pleiotropic influence of the gene ( $p)$ on all these characters could be demonstrated by the study of white mutants of the black-seeded cultivars PI226895 and Aiguillon. Some discrepancies could appear between ranking for Pythium susceptibilities in experiments with $10 \mathrm{~d}$ incubation at $11^{\circ} \mathrm{C}$ and ranking for these biochemical characters. They could be linked to differences in physiological ability to germinate and grow at low temperatures. These differences were also studied: the best cultivar found for this character was Vernandon. Integration of control measures has been discussed. Even the low levels of resistance which can be bred for white-seeded lines can be improved by seed treatment with low amounts of fungicides. White-seeded cultivars are preferred by the snap bean canning industry. The water in the snap bean cans is blue-gray with black-seed cultivars. Light-buff colored resistant lines can be bred, resulting in a less unpleasant water coloration in the cans.

bean $=$ Phaseolus vulgaris $L /$ germination $/$ cold soils $/$ Pythium

* Correspondance et tirés à part. 


\section{INTRODUCTION}

Aussi bien l'industriel, soucieux d'allonger la période de fonctionnement de l'usine, que le maraîcher qui désire profiter de la plus-value apportée à sa récolte par la précocité, souhaitent débuter le plus tôt possible la récolte de haricots en filets fins, mange-tout ou flageolets verts.

De là, la tentation d'avancer la date des semis printaniers, au risque d'observer des germinations irrégulières ou nulles.

La tradition paysanne de chaque région indique la date où l'on peut tenter un premier semis de haricots : c'est la Saint-Joseph (19 mars) pour le midi de la France (Vaucluse, Bouchesdu-Rhône).

On observe d'importantes différences entre variétés de haricot pour l'aptitude à la réussite de ces semis précoces.

Les causes des manques à la levée sont d'une double nature : mauvaise germination à basse température, mais aussi envahissement du grain entier ou de la radicule en début de croissance par des Pythium hôtes du sol - en admettant bien sûr que n'intervienne pas la mouche des semis (Phorbia platura, autrefois Hylemia cilicrura), ou que des traitements insecticides adéquats aient été réalisés (Schvester et Rives, 1957).

Les travaux que nous résumerons ci-dessous ont eu pour but de mieux connaître les facteurs qui rendent les variétés de haricot plus ou moins aptes à une bonne germination en semis printanier précoce.

\section{MATÉRIEL ET MÉTHODES}

\section{Génotypes de haricot}

Nous avons utilisé, d'une part des variétés représentatives des divers types de haricots cultivés en France pour la production de filets fins, mange-tout, grains à écosser ou flageolets verts, d'autres part des lignées que la littérature américaine (Dickson et Boettger, 1975; Hagedorn et Rand, 1978), ou P Touzard (ets Clause, comm pers) nous signalaient pour la résistance aux Pythium. Nous y avons ajouté des lignées sélectionnées par l'un d'entre nous à l'INRA-Versailles dans la descendance de croisements Phaseolus vulgaris $\times P$ coccineus (sl) réalisés par $\mathrm{H}$ Bannerot (INRA-Versailles). La tradition des maraîchers de la zone Vaucluse-Bouches-du-Rhône nous a, de plus, indiqué les variétés Saint Marcellin et Noir Hâtif de
Saint-Rémy réputées pour leur bonne réussite en semis précoces.

\section{Souches de Pythium}

Nous avons tout d'abord étudié une collection de 20 souches isolées de végétaux attaqués, ou directement du sol (Messiaen et al, 1977). Leurs caractères morphologiques, et des expériences préliminaires de contamination sur 4 variétés nous ont permis de les classer de la façon suivante (les isolats pouvant être considérés comme très agressifs sont soulignés de 2 traits, les moyennement agressifs d'un trait, les faiblement agressifs non soulignés) :

- Pythium ultimum Trow: souches $\stackrel{\mathrm{P}_{1}}{=}, \underline{\mathrm{P}_{2}}, \stackrel{\mathrm{P}_{3}}{=}, \underline{\mathrm{P}_{4}}, \underline{\mathrm{P}_{5}}$, - Pythium sylvaticum Campb et Hend: $\mathrm{P}_{7}, \underline{\mathrm{P}_{8}}, \underline{\mathrm{P}_{12}}$ $\underline{P_{13}}, \underline{P_{17}}, \underline{\underline{P_{20}}}$,

- souches non déterminées de type palmé-coralloïde (Messiaen et al, 1977) : $P_{6}, P_{11}, P_{16}, P_{18}$,

- souches ne rentrant dans aucune des catégories précédentes: $\underline{P}_{15}, P_{19}$

Pour la suite de nos expériences, nous avons employé les souches $P_{2}, P_{12}$ et $P_{16}$, représentatives des 3 degrés d'agressivité.

\section{Conditions de germination et d'inoculation}

Nous avons testé sur plusieurs variétés de haricot, présumées plus ou moins résistantes, diverses méthodes d'inoculation, comme la confrontation directe des grains et du champignon sur boîtes de Petri de gélose non nutritive, ou le semis en pot sur vermiculite imbibée de solution de Knop, les grains étant posés sur un disque de gélose non nutritive colonisé par le Pythium, et recouverts de $2 \mathrm{~cm}$ de vermiculite : nous retiendrons surtout les résultats de cette dernière méthode. Les pots ainsi préparés étaient incubés $10 \mathrm{j}$ à $11^{\circ} \mathrm{C}$, puis placés à $20^{\circ} \mathrm{C}$. La germination était notée au $20^{\mathrm{e}}$ j suivant une échelle de 0 à 15 tenant à la fois compte du développement végétatif des plantules, et des lésions dues au Pythium sur radicules, racines et hypocotyles.

\section{Dosages chimiques}

Parmi les dosages que nous avons pratiqués, les résultats les plus intéressants concernaient les exsudations des grains en début de germination, nutritives pour les Pythium, et la teneur des grains en leucoanthocyanes, présumées fongistatiques.

Les exsudats solubles totaux de grains trempés dans une quantité d'eau double de leur poids pendant $24 \mathrm{~h}$ ont été évalués en degrés Brix mesurés au réfractomètre. 
Les résultats obtenus avec cet appareil ont été complétés par des dosages de sucres (réducteurs et totaux) avec un appareil Technicon, et d'azote total suivant la méthode de Kjeldahl.

Pour le dosage des leuco-anthocyanes, les grains ont été trempés dans une quantité d'éthanol à $50^{\circ} \mathrm{cor}-$ respondant au double de leur poids. Les extraits obtenus, dilués 10 fois dans l'éthanol-chlorhydrique ( $2 \%$ en volume d'HCl pur $12 \mathrm{~N}$ ) peuvent être directement examinés au spectrophotomètre à $550 \mathrm{~nm}$ (anthocyanes) et $340 \mathrm{~nm}$ (flavonoïdes). Mais ces 2 mesures ne donnent pas de résultats intéressants en liaison avec la résistance variétale aux Pythium, ce sont les leucoanthocyanes qui nous ont paru les plus impliquées dans ce phénomène.

Elles sont révélées par une méthode due à Lebreton et al (1967). Deux millilitres de l'extrait obtenu par trempage des grains $24 \mathrm{~h}$ dans l'alcool à $50^{\circ}$ sont dilués dans $20 \mathrm{ml}$ d'un mélange eau $+\mathrm{HCl}+$ éthanol (42-2-44). On filtre sur papier. On ajoute à $5 \mathrm{ml}$ de filtrat $15 \mathrm{ml}$ d'un mélange $60 / 40$ butanol primaire $+\mathrm{HCl}$ pur. On teste à $550 \mathrm{~nm}$ au spectrophotomètre (lecture $\left.D_{0}\right)$.

Le mélange est chauffé au bain-marie bouillant $15 \mathrm{~min}$, puis rapidement refroidi dans la glace. On fait une seconde lecture à $550 \mathrm{~nm}$ (lecture $D_{1}$ ). La teneur de l'extrait, exprimée en leucocyanidine, est donnée dans les conditions de macération et de prise d'essai décrites ci-dessus par la formule $\left(D_{1}-D_{0}\right) \times 1040$.

\section{Reproductibilité des résultats}

Les résultats des confrontations haricot/Pythium sur vermiculite avec incubation à $11^{\circ} \mathrm{C}$ sont très reproductibles d'une expérience à l'autre, et homogènes entre les pots de 6 grains constituant les répétitions d'une même expérience, pour les notes les plus faibles $(0$, sensibilité totale) ou les plus élevées (15, bonne résistance). L'étendue de leur variation est de l'ordre de \pm 2 pour les notes intermédiaires. La variabilité des dosages du degré Brix des exsudats est de l'ordre de $10 \%$ pour le même lot de semences d'une expérience à l'autre, celle des dosages chimiques (sucres, azote, leuco-anthocyanes) de l'ordre de 15\%. Les mesures d'épaisseur de téguments des grains (coupes au rasoir à main examinées au microscope) font apparaître une variation de l'ordre de $15 \%$ d'un grain à l'autre dans un même lot de semences.

\section{RÉSULTATS}

\section{Sensibilités variétales aux Pythium}

Nous en donnerons comme exemple au tableau I les résultats d'un essai mettant en jeu 38 variétés et 3 souches de Pythium.

Les variétés PI226895, Pl163583 et PI109859 se classent comme les plus résistantes. D'une façon générale, malgré une importante variation dans chaque catégorie, les variétés à grain noir (ou rouge, dans le cas de Pl109859) sont les moins sensibles, suivies par celles à grain beige ou panaché. Parmi les variétés à grain blanc, les lignées sélectionnées pour la résistance aux $P y$ thium ne manifestent celle-ci que vis-à-vis de la souche la moins agressive. On remarquera l'extrême sensibilité des flageolets verts.

En dernière colonne du tableau, nous avons mentionné les résultats (en pourcentage de survivants) d'un semis du 21 mars à Eyragues (Bouches-du-Rhône), en conditions de plein champ assez défavorables (sol humide et tassé). Nous avons calculé la corrélation entre ce résultat et la moyenne des notes obtenues avec les souches $P_{12}$ et $P_{16}$. Elle se révèle élevée et significative $(R=0,85)$.

\section{Hérédité de la résistance de PI226895}

Ayant réalisé le croisement entre la variété la plus résistante, $\mathrm{Pl} 226985$ et Elsa, flageolet vert extrêmement sensible, nous avons pratiqué des tests de résistance aux Pythium au cours des générations suivantes :

\section{Embryon $F_{1}$ sous tégument $F_{0}$}

Le résultat est très différent suivant le sens du croisement (tableau II).

\section{Embryon $F_{2}$ sous tégument $F_{1}$}

À cette génération, les grains sont tous panachés noir sur fond beige. L'avantage "parent maternel PI226895" s'efface presque complètement (tableau III).

\section{Embryon $F_{3}$ sous tégument $F_{2}$}

Nous avons, à partir de cette génération, confondu les 2 sens de croisement et regroupé les lignées en 3 classes :

$\mathrm{S}$, "sensibles" - notes de 0,0 à 4,9

I, «intermédiaires» - notes de 5 à 9,9

$R$, «résistantes» - notes de 10 à 15 .

Les résultats, pour 150 lignées, sont exprimés au tableau IV.

Ces résultats nous indiquent que l'on peut trouver d'aussi bonnes résistances chez les li- 
Tableau I. Sensibilités variétales vis-à-vis des Pythium: notes de 0 à 15 .

\begin{tabular}{|c|c|c|c|c|c|c|}
\hline Variété & Obtenteur & $\begin{array}{l}\text { Coloration } \\
\text { du grain }\end{array}$ & $\begin{array}{l}\text { Souche } P_{2} \\
\text { (ultimum) }\end{array}$ & $\begin{array}{l}\text { Souche } P_{12} \\
\text { (sylvaticum) }\end{array}$ & $\begin{array}{c}\text { Souche } P_{16} \\
\text { (palmé-coralloïde) }\end{array}$ & $\begin{array}{l}\% \text { Survivance } \\
\text { au champ } \\
\text { semis } 21 \text { mars }\end{array}$ \\
\hline
\end{tabular}

Géniteurs, ou lignées sélectionnées pour la résistance

\section{$\mathrm{PI} 226895$ \\ PI163583 \\ Pl109859}

St Marcellin

$\mathrm{BF} 11$

6021

6716

6717

BPy 153

BPy 157

764B

BPy 10

RRR77

5062

RRR83

6783

\section{Noir \\ 12,4}

Noir

Rouge

Noir

Beige

Blanc

Blanc

Blanc

Panaché

Panaché

(c)

Boettger

(c)

Hagedorn

Boettger

Hagedorn

Dickson

Blanc

Blanc

Blanc

Blanc

Blanc

Blanc

8,3

6,5

0,0

0,0

0,0

0,0

1,8

1,5

0,0

0,0

0,0

0,0

0,0

0,0

0,0

Variétés pour filets fins

Aiguillon
César

Noir hatif SR

Êtendard

Centurion

Gautier

Gautier

(b)

Gautier

Gautier
Noir

Panaché Noir

Panaché

Panaché

1,5
0,2
0,0
0,0
0,0

Variétés mange-tout

Contender

Irago

Vaillant

DP

Brelan

Gautier

INRA

Arly

Caillard

Caillard

$\begin{array}{cc}\text { Beige } & 1,5 \\ \text { Noir } & 0,0 \\ \text { Blanc } & 0,0 \\ \text { Blanc } & 0,0 \\ \text { Blanc } & 0,0\end{array}$

Variétés pour grains blancs à écosser

Blason Gautier Blanc

Michelite DP Blanc

Lingot DP Blanc

Coco NB précoce DP

Coco Sophie

Gautier

Blanc

Blanc

Michelet

DP

Blanc

Blanc

$\begin{array}{ll}0,0 & 0,0 \\ 0,0 & 0,0 \\ 0,0 & 0,0 \\ 0,0 & 0,0 \\ 0,0 & 0,0 \\ 0,0 & 0,0 \\ 0,0 & 0,0\end{array}$

10,6

0,0

0,0

0,0

0,0

6,0

2,4

0,0

0,0

11,3

15,0

10,0
6,6

0,0

0,0

0,0
0,7

0,4

0,0

0,0

0,0
0,0

0,0

0,0

15,0

15,0

15,0

15,0

15,0

15,0

15,0

15,0

15,0

15,0

9,1

8,0

8,0

7,2

7,0

5,3

64

51

33

44

53

22

31

10

39

24

18

13

$-$

9

--

5

Variétés flageolets verts

\begin{tabular}{lclllll} 
Manacor & Clause & Vert pâle & 0,0 & 0,0 & 1,5 & - \\
Vernor & Séminor & Vert pâle & 0,0 & 0,0 & 1,0 & - \\
Ardent & INRA & Vert pâle & 0,0 & 0,0 & 0,5 & 1 \\
Elsa & INRA & Vert pâle & 0,0 & 0,0 & 0,4 & - \\
Vernel & Vilmorin & Vert pâle & 0,0 & 0,0 & 0,2 & \\
\hline
\end{tabular}

(a) Variétés signalées comme résistantes à Rhizoctonia solani par Zaumeyer, repérées par $\mathrm{P}$ Touzard (communication personnelle) pour leur tolérance aux Pythium, (b) : variétés traditionnelles des environs de St Rémy de Provence; (c) : lignées sélectionnées à partir de l'hybride Phaseolus vulgaris x P coccineus sensu lato obtenu par H Bannerot (INRA - Versailles). DP : variétés dans le domaine public. 
Tableau II. Comportement vis-à-vis des Pythium des grains $F_{1}$ des croisements entre Elsa et PI226895.

Notes de survie des plantules (0-15)

\begin{tabular}{llrl}
\hline Grains $F_{1}$ & $P_{2}$ & $P_{12}$ & $P_{16}$ \\
Elsa x PI226895 & 0,0 & 0,9 & 12,0 \\
PI226895 x Elsa & 8,9 & 13,1 & 15,0 \\
& & & \\
\hline
\end{tabular}

Tableau III. Comportement des grains $\mathrm{F}_{2}$.

Notes de survie des plantules (0-15)

\begin{tabular}{llll}
\hline Grains $F_{2}$ & $P_{2}$ & $P_{12}$ & $P_{16}$ \\
Elsa x PI226895 & 0,3 & 6,3 & 15,0 \\
PI226895 x Elsa & 1,4 & 8,2 & 15,0 \\
\hline
\end{tabular}

gnées à tégument beige ou panaché que chez celles à grain noir. Mais, dans le cas présent, la coloration panachée est infixable, puisqu'elle correspond à des plantes mères hétérozygotes pour les allèles $C$ et $c$ du gène gouvernant l'intensité de coloration des grains (Prakken, 1972). Par contre, les lignées à grain blanc (allèle récessif $p$ du gène $P$ indispensable à l'expression de toute coloration) sont nettement défavorisées vis-à-vis des souches $P_{2}$ et $P_{12}$.

\section{Embryon $F_{7}$ sous tégument $F_{6}$}

Nous avons examiné, pour cette génération, le comportement de 190 lignées issues de «filiation unipare" (ou "SSD»), dont le comportement visà-vis des souches $P_{2}$ et $P_{12}$ en fonction de la coloration est détaillé au tableau $\mathrm{V}$.
L'absence de lignées à grain blanc résistantes ou intermédiaires par leur comportement vis-àvis de $P_{12}$ (moyennement agressive) ou $P_{2}$ (très agressive), confirme l'effet défavorable de l'allèle $\mathrm{p}$, et le faible nombre de lignées à grain panaché le caractère «infixable" de ce caractère.

On remarquera aussi, parmi les lignées à grain noir ou beige, le faible effectif de lignées à comportement intermédiaire, que l'un d'entre nous (Ginoux, 1981) avait attribué à la présence chez Pl226895 d'un gène dominant de résistance Rpy.

Avec le recul des années et l'expérience aux Antilles, dans des sols contaminés par Pythium aphanidermatum, de l'échec d'un essai de sélection de flageolets noirs, repérés à la germination par leurs cotylédons incolores, nous pensons aujourd'hui que ce gène Rpy n'était en fait que l'allèle dominant du gène récessif conditionnant le caractère "flageolet vert" chez Elsa, et qui s'exprime de façon moins évidente chez les génotypes à grain coloré. II conserve cependant certains de ses effets pléiotropiques : fanaison prématurée des plantes en fin de végétation, avec comme conséquence une polymérisation imparfaite de l'amidon des grains, cotylédons blancs à la germination - ainsi sans doute qu'une sensibilité particulière aux Pythium.

L'expérience relatée au tableau VI aurait été plus complète si elle avait fait intervenir aussi l'isolat peu agressif $P_{16}$, qui aurait permis de distinguer parmi les lignées à tégument incolore les «flageolets» des «non-flageolets».

Parallèlement à cette étude en filiation unipare, avait été conduite une sélection généalogique pour la résistance, avec tri en $F_{3}$ et $F_{7}$. Nous avions ainsi obtenu 3 lignées à grain beige notées 15 vis-à-vis de $P_{2}$ et $P_{12}$, et 2 lignées à grain blanc notées 0 vis-à-vis de $P_{2}$, mais respectivement 8,4 et 6,3 vis-à-vis de $P_{12}$ (nous re-

Tableau IV. Classement des lignées $F_{3}$ pour la résistance.

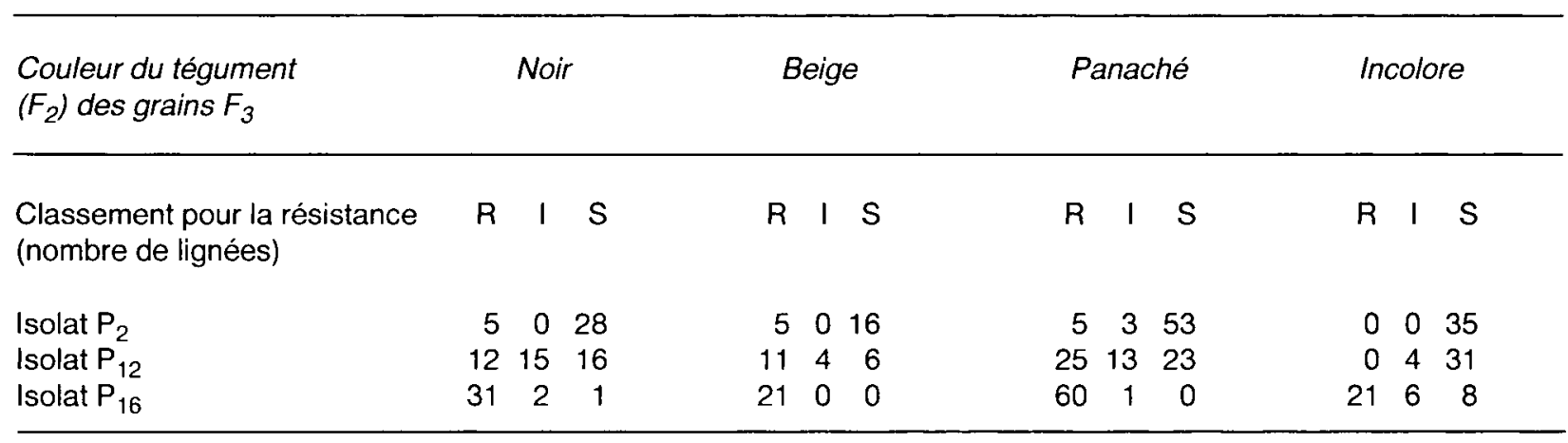


Tableau V. Classement des lignées $F_{7}$ pour la résistance.

\begin{tabular}{|c|c|c|c|c|}
\hline $\begin{array}{l}\text { Couleur du tégument } \\
\left(F_{6}\right) \text { de } 190 \text { lignées } F_{7}\end{array}$ & Noir & Beige & Panaché & Incolore \\
\hline $\begin{array}{l}\text { Classement pour la résistance } \\
\text { (Nombre de lignées) }\end{array}$ & $R$ I $S$ & $R \mid S$ & $R \mid S$ & $R \mid S$ \\
\hline $\begin{array}{l}\text { Isolat } P_{2} \\
\text { Isolat } P_{12}\end{array}$ & $\begin{array}{lll}22 & 0 & 24 \\
20 & 2 & 24\end{array}$ & $\begin{array}{lll}16 & 1 & 25 \\
19 & 1 & 22\end{array}$ & $\begin{array}{lll}5 & 0 & 1 \\
6 & 0 & 0\end{array}$ & $\begin{array}{lll}0 & 0 & 96 \\
0 & 0 & 96\end{array}$ \\
\hline
\end{tabular}

trouverons ces lignées A27.1.4.1 et A54.1.2.3 dans le paragraphe Interaction résistance/ fongicides).

\section{Étude de mutants à grains blancs dans les variétés PI226895 et Aiguillon}

Nous avons eu la chance de trouver, dans des lots de semences de chacune de ces 2 variétés, 1 grain blanc, parmi les grains noirs qui les caractérisent.

Dans les 2 cas, la descendance de ces grains s'est avérée homogène, et les plantes morphologiquement semblables à la variété d'origine. Les hybrides $F_{1}$ avec PI226895 et Aiguillon ont été eux aussi semblables à la variété d'origine, sans montrer aucune hétérosis, et la ségrégation des téguments $F_{2}$ de type 3/4-1/4.

II s'agit donc probablement dans les 2 cas de mutations récessives du gène $P$.

Nous avons obtenu les résultats suivants en confrontant les mutants et les variétés d'origine aux isolats $P_{2}, P_{12}$ et $P_{16}$ (tableau $V I$ ).

Cette observation confirme donc l'effet défavorable de l'allèle $p$, et nous permettra d'étudier ses effets pléïotropiques (voir ci-dessous).

\section{Analyse des facteurs de sensibilité aux Pythium}

Outre l'effet du gène $p$ chez les grains blancs, il existe d'autres facteurs de sensibilité aux $P y$ thium, puisque l'on trouve chez les grains noirs des variétés de sensibilité très différente. Nous avons cherché à les identifier en utilisant une collection réduite, représentant divers types de comportement :
PI226895 : noir résistant,

Saint-Marcellin : noir peu sensible,

Noir Hâtif : noir assez sensible,

Contender : beige peu sensible,

BF11 : beige moyennement sensible,

Michelite : blanc assez sensible,

Elsa : flageolet vert très sensible.

La confrontation directe des grains de ces variétés avec les Pythium cultivés en boîtes de Petri de gélose non nutritive avait fait apparaître une stimulation mycélienne au voisinage des grains, localisée à la zone hile-micropyle, et grossièrement proportionnelle à la sensibilité variétale, sauf chez Elsa : dans ce cas la stimulation se manifestait tout autour du grain, rapidement envahi par le mycélium.

Cela laissait supposer, à partir du grain de haricot, une exsudation de substances stimulant les Pythium, éventuellement contrebalancée, pour les moins sensibles, par des substances fongistatiques, parmi lesquelles nous avons choisi, à la suite de dosages peu convaincants concernant les substances colorées, de rechercher les leuco-anthocyanes.

Tableau VI. Comportement vis-à-vis des Pythium de mutants à grains blancs de variétés à grains noirs.

Isolats de Notes de survie des plantules Pythium

\begin{tabular}{lcccc}
\hline & PI226895 & $\begin{array}{c}\text { Pl225895 } \\
\text { blanc }\end{array}$ & Aiguillon & $\begin{array}{c}\text { Aiguillon } \\
\text { blanc }\end{array}$ \\
$\mathrm{P}_{2}$ & 11,0 & 0,0 & 4,0 & 0,0 \\
$\mathrm{P}_{1}$ & 15,0 & 4,5 & 8,0 & 0,0 \\
$\mathrm{P}_{16}$ & 15,0 & 15,0 & 15,0 & 13,0 \\
\hline
\end{tabular}


L'usage des procédés décrits au paragraphe Matériel et méthodes nous a permis d'obtenir les résultats décrits au tableau VII, en y ajoutant l'épaisseur du tégument mesurée en coupe au microscope.

Nous avions comparé de plus à cette petite collection les mutants à grains blancs mentionnés ci-dessus, avec leurs variétés d'origine (tableau VIII).

Des expériences complémentaires (Ginoux, 1981), que nous ne détaillerons pas ici, ont mis en comparaison des grains intacts ou dépouillés de leur tégument, des grains témoins et des grains enduits de vernis imperméable sur la zone hile-micropyle. Elles confirment l'influence prédominante du tégument sur l'exsudation, et que celle-ci se produit principalement au niveau de la zone hile-micropyle, sauf chez Elsa.

D'autres expériences ont permis de démontrer le caractère "passif» de la résistance de type "PI226895". En effet, si dans le dispositif habituel d'essai sur vermiculite 1, 2 ou 3 des grains de Pl226895 sont remplacés par des aéléments contaminants" constitués, soit par des grains d'Elsa, soit par des grains de Pl226895 blessés, tués par la chaleur ou récoltés à l'état immature, on assiste à une baisse progressive de la note obtenue sur PI226895 de 15 à 0 avec 3 éléments contaminants, en présence d'une souche moyennement agressive.

Au contraire, en compagnie de 5 grains de $\mathrm{Pl} 225895$, en confrontation avec une souche peu agressive, un grain d'Elsa donne une plantule notée 15 (contre 0,4 pour 6 grains d'Elsa).

Ces observations donnent à réfléchir sur la plantation en poquets, souvent pratiquée par les amateurs dans l'espoir d'améliorer l'émergence de leurs semis. L'effet espéré ne sera obtenu sans doute que si la variété n'est pas trop sensible aux attaques de Pythium, tous les grains étant indemnes de blessures au niveau du tégument ou du germe, et de bonne énergie germinative.

\section{Aptitude à la germination à basse température}

Si nous revenons au tableau VII, nous remarquerons que la variété Michelite, particulièrement défavorisée par ses exsudations sucrées et azotées et la minceur de son tégument, de-

Tableau VII. Caractéristiques biochimiques et histologiques de 7 variétés.

\begin{tabular}{lcccccr}
\hline Variétés & $\begin{array}{c}\text { Degré BRIX } \\
\text { del'exsudat }\end{array}$ & $\begin{array}{c}\text { Sucres } \\
\text { totaux } g / l\end{array}$ & $\begin{array}{c}\text { Sucres réducteurs } \\
(g / l)\end{array}$ & $\begin{array}{c}\text { Azote } \\
(\mathrm{mg} / \mathrm{l})\end{array}$ & $\begin{array}{c}\text { Leuco-anthocyanes } \\
(\mathrm{mg} / \mathrm{l})\end{array}$ & $\begin{array}{c}\text { Epaisseur du } \\
\text { tégument }(\mu)\end{array}$ \\
\hline Pi226895 & 1,3 & 0,49 & 0,19 & 266 & 832 & 213 \\
St Marcellin & 5,7 & 2,63 & 0,79 & 100 & 190 & 196 \\
Noir Hâtif & 9,3 & 2,71 & 0,95 & 361 & 286 & 217 \\
Contender & 4,6 & 0,60 & 0,40 & 245 & 645 & 136 \\
BF11 & 5,6 & 3,28 & 1,08 & 493 & 452 & 104 \\
Michelite & 17,6 & 7,88 & 1,26 & 1064 & 130 & 90 \\
Elsa & 11,5 & 5,20 & 2,68 & 355 & 21 & 134 \\
\hline
\end{tabular}

Tableau VIII. Caractéristiques biochimiques et histologiques de mutants à grain blanc.

\begin{tabular}{|c|c|c|c|c|c|c|}
\hline Variétés & $B R I X$ & $\begin{array}{l}\text { Sucres totaux } \\
\qquad(g / l)\end{array}$ & $\begin{array}{l}\text { Sucres réducteurs } \\
\qquad(g / l)\end{array}$ & $\begin{array}{l}\text { Azote } \\
(m g / l)\end{array}$ & $\begin{array}{l}\text { Leuco-anthocyanes } \\
\text { (mg/l) }\end{array}$ & $\begin{array}{l}\text { Épaisseur du } \\
\text { tégument }(\mu)\end{array}$ \\
\hline PI226895 & 1,3 & 0,61 & 0,23 & 266 & 832 & 213 \\
\hline $\begin{array}{l}\text { Pl226895 } \\
\text { blanc }\end{array}$ & 5,5 & 2,40 & 0,93 & 179 & 282 & 161 \\
\hline Aiguillon & 2,5 & 0,9 & 0,67 & 400 & 289 & 172 \\
\hline $\begin{array}{l}\text { Aiguillon } \\
\text { blanc }\end{array}$ & 7,0 & 1,96 & 1,16 & 479 & 53 & 153 \\
\hline
\end{tabular}


vrait se comporter encore plus mal que Elsa. Or ce n'est pas le cas, aussi bien au laboratoire qu'au champ.

Nous avons pensé que cette anomalie pouvait trouver son explication dans une meilleure aptitude à la germination de Michelite pendant l'incubation à $11^{\circ} \mathrm{C}$.

Cela nous a entraînés à étudier, en l'absence de Pythium, l'aptitude à germer à basse température chez le haricot.

Pour cette étude, nous avons ajouté à quelques variétés de la collection étudiée précédemment les lignées ou variétés suivantes:

- S412, S414, S419 et S435 fournies par Boettger,

- Geneva 1.161 (Dickson 1972),

- Comtesse de Chambord, signalée comme germant bien à basse température par Hardwick (1971).

- Vernandon (Caillard) et Étendard (Gautier), remarqués par les producteurs comme réussissant bien en semis précoce.

Nous mentionnerons ci-dessous les résultats d'un essai de germination sur boîte de Petri à $11^{\circ} \mathrm{C}$, et ceux d'un essai sur vermiculite en enceinte climatisée et éclairée à $10^{\circ}-12^{\circ} \mathrm{C}$, noté à l'aide d'un indice attribuant à chaque grain ou plantule, au bout de $20 \mathrm{j}$ les notes suivantes:

0 : non émergé,

0,333 : crosse pointée,

0,666 : crosse développée,

$1,000: 2$ premières feuilles étalées.

La somme de ces notes, pour 100 grains semés, donnant un indice de 0 à 100 (le tout en l'absence de Pythium, bien entendu), voir tableau IX.

Ces résultats confirment ce que nous soupçonnions précédemment : Michelite germe beaucoup mieux que Elsa à basse température.

Conservées plus longtemps dans la même enceinte climatisée $\left(10^{\circ}-12^{\circ} \mathrm{C}, 4000\right.$ lux au niveau des plantes) les plantules de 5 des variétés ci-dessus se distinguent par l'aspect de leur feuillage au $35 \mathrm{e} \mathrm{j}$ : chlorosé et nain pour Michelite et Comtesse de Chambord, chlorosé mais normal pour $\mathrm{Pl} 226895$, légèrement chlorosé pour BF11, normalement vert et développé pour Vernandon.

Cette variété (à grain noir pour filets fins) se détache donc de la plupart des autres pour son aptitude à germer et à croître à basse température, ce qui nous a amenés à la comparer à
Pl226895 pour la résistance aux Pythium et les caractéristiques biochimiques et histologiques (voir tableau X).

Le cultivar Vernandon, par ses caractéristiques biochimiques et histologiques, laisserait donc prévoir un comportement plus mauvais visà-vis des Pythium que PI226895, ce qui est largement compensé par sa meilleure aptitude à la germination à basse température.

Nous avons réalisé le croisement (Vernandon $x \mathrm{Pl} 26895)$ et son réciproque. De l'étude de 310 lignées $F_{3}$, nous pouvons conclure à une hérédité récessive de l'aptitude à germer et croître à basse température, compliquée par l'intervention d'un facteur cytoplasmique : 10 lignées adaptées au froid sur 130 avec Vernandon comme parent femelle, 1 sur 180 avec PI226895.

\section{INTÉGRATION DES MOYENS DE LUTTE CONTRE LA MAUVAISE GERMINATION EN SOL FROID}

Rappelons tout d'abord que tous les sols ne sont pas contaminés au même point par des Pythium

Tableau IX. Aptitudes variétales à la germination et à la croissance à basse température.

\begin{tabular}{lrc}
\hline & $\begin{array}{r}\text { Essai } \\
\text { in vitro }\end{array}$ & $\begin{array}{c}\text { Essai sur } \\
\text { vermiculite } \\
\text { à } 11^{\circ} \mathrm{C} \\
(\text { (G50te de } 0-15)\end{array}$ \\
\hline Vernandon & & \\
Michelite & 7,0 & 79,0 \\
Comtesse de Chambord & 6,0 & 73,3 \\
BF.11 & 7,5 & 54,7 \\
Étendard & 9,0 & 68,0 \\
St Marcellin & 7,5 & 35,3 \\
S.412 & 10,5 & 35,0 \\
Noir Hâtif & 10,5 & 34,0 \\
S.435 & 8,5 & 32,7 \\
Irago & 9,0 & 31,7 \\
S.414 & 8,5 & 23,6 \\
PI.163583 & 11,5 & 23,3 \\
Geneva 1.161 & 12,5 & 16,7 \\
Elsa & 11,5 & 13,3 \\
S.417 & 8,5 & 6,6 \\
Contender & 10,5 & 6,0 \\
PI.109859 & 11,5 & 5,3 \\
PI.226895 & 12,5 & 2,0 \\
& 11,5 & 0,0 \\
\hline
\end{tabular}

- G50 : nombre de jours pour atteindre 50\% de germination. 
agressifs, et que les précédents culturaux exercent une influence importante (Messiaen et al, 1977).

Dans les sols provenant de prairies de graminées prédominent les souches de type «palmécoralloïde", dans les sols cultivés en plantes annuelles peuvent prédominer les Pythium sylvaticum et ultimum. Mais l'estimation globale du pouvoir pathogène de ces microflores indique une agressivité moindre pour les précédents "céréales" que pour les précédents “légumineuses". Ces observations peuvent nous donner une indication sur le risque encouru en cas de semis précoce d'une variété à grain blanc ou flageolet vert.

On ne peut manquer par ailleurs de nous faire observer que l'usage de fongicides en traitement de semences peut enlever tout intérêt aux travaux exposés ci-dessus ! Pour approfondir ce point de vue, nous avons expérimenté l'enrobage des grains avec des fongicides d'usage courant (Thirame, Captane) ou expérimentaux (éthylphosphite d'aluminium, prothiocarbe, curzate, thiophanate-méthyl, métalaxyl) sur des grains de Noir Hâtif (moyennement sensible) et Elsa (très sensible). Alors que Noir Hâtif supporte sans phytotoxicité des doses allant jusqu'à $5 \mathrm{~g}$ de $\mathrm{ma} /$ $\mathrm{kg}$ de grains, Elsa manifeste une phytotoxicité importante à $2,5 \mathrm{~g} / \mathrm{kg}$ pour tous les produits, la dose bien supportée en l'absence de Pythium ne dépassant pas $1,25 \mathrm{~g} / \mathrm{kg}$ de grains.

Nous avons ensuite confronté sur vermiculite avec l'isolat $\mathrm{P} 2$, incubation à $11^{\circ} \mathrm{C}$, des grains d'Elsa, et des lignées à grains blanc sélectionnées pour une résistance d'un niveau assez faible, A27.1.4.1 et A54.1.2.3, avec ou sans enrobage fongicide.

Tableau $X$. Caractéristiques comparées de "PI226895" et "Vernandon».

\section{Vernandon Pl226895}

\begin{tabular}{lcc}
$\begin{array}{l}\text { Note Pythium P2 } \\
\text { (vermiculite - P11 }\end{array}$ & 11,9 & 12,4 \\
$11^{\circ}-20^{\circ}$ ) P16 & 15,0 & 15,0 \\
& 15,0 & 15,0 \\
Épaisseur du & & \\
tégument $\mu$ & 132 & 213 \\
Degré Brix & 3,2 & 1,3 \\
& & \\
Sucres totaux (g/l) & 0,73 & 0,49 \\
Sucres réducteurs $(\mathrm{g} / \mathrm{l})$ & 0,29 & 0,19 \\
Leuco-anthocyanes $(\mathrm{mg} / \mathrm{l})$ & 801 & 832 \\
\hline
\end{tabular}

Le tableau XI exprime les résultats.

La "résistance», même faible, des 2 lignées "A" semble donc potentialiser l'effet des doses faibles de fongicides.

\section{DISCUSSION ET CONCLUSION}

Sans qu'il soit nécessaire de recourir aux croisements interspécifiques, il existe déjà chez Phaseolus vulgaris une variabilité importante pour la résistance aux Pythium et l'aptitude à germer et croître à basse température.

Ce sera chez des variétés à grains colorés en noir, rouge ou beige que l'on trouvera les moindres sensibilités aux Pythium. L'effet pléiotropique qu'exerce le gène $p$ sur la couleur du grain, mais aussi sur l'épaisseur et la perméabilité du tégument et sa teneur en leucoanthocyanes rend peu probable l'obtention de variétés à grain blanc hautement résistantes aux Pythium les plus agressifs. Un faible niveau de résistance, sélectionnable avec une souche de type "palmé-coralloïde" permettrait cependant une meilleure émergence en conditions moyennes, et une meilleure efficacité de l'enrobage fongicide en conditions limites.

On pourraît à première vue renoncer à l'emploi de variétés à grains blancs pour la production précoce de filets fins ou de mange-tout. Cependant l'industrie les préfère, car les gousses des variétés à grains colorés contiennent des anthocyanes colorant en gris-bleu l'eau des boîtes.

Des essais de stérilisation réalisés avec des haricots verts provenant de lignées issues de (PI225895 x Elsa) ont montré que, si les jus de

Tableau XI. Interaction entre enrobage fongicide et résistance aux Pythium (notes de 0-15, souche P2).

\begin{tabular}{|c|c|c|c|c|c|c|}
\hline \multirow[t]{3}{*}{ Lignées } & \multicolumn{6}{|c|}{ Fongicides (gma/kg de grains) } \\
\hline & \multicolumn{3}{|c|}{ Metalaxyl } & \multicolumn{3}{|c|}{ Captane } \\
\hline & 0,16 & 0,32 & 0,63 & 0,16 & 0,32 & 0,63 \\
\hline A27.1.4.1 & 14,4 & 15,0 & 15,0 & 12,3 & 15,0 & 15,0 \\
\hline A54.1.2.3 & 9,3 & 10,5 & 15,0 & 4,1 & 5,4 & 15,0 \\
\hline Elsa & 0,3 & 4,8 & 15,0 & 0,0 & 0,0 & 15,0 \\
\hline
\end{tabular}

Les témoins non traités étaient notés 0 dans les 3 cas. 
cuisson présentaient une coloration franchement désagréable pour les lignées à grains noirs, ils n'étaient que légèrement plus opaques que ceux des lignées à grain blanc dans le cas de celles à grain beige.

Le cas des flageolets verts est particulier : la qualité même du produit est liée à un caractère génétique à effet pléiotropique qui sensibilise aux Pythium le grain en germination. La prudence restera sans doute toujours nécessaire dans le choix de la date de semis pour ce type variétal, auquel on réservera si possible les précédents les moins favorables aux Pythium, et un enrobage fongicide à une dose tenant compte des risques de phytotoxicité.

\section{REMERCIEMENTS}

Les recherches exposées dans cet article ont été effectuées dans les laboratoires de la société Gautier \& fils (13630 Eyragues), qui les a encouragées et rendues matériellement possibles au cours des années 1978-1981.

Nous remercions également :

- Mme le professeur N Paris (physiologie végétale USTL Montpellier) pour ses précieux conseils à l'un d'entre nous dans le cadre de la rédaction d'une thèse;

- les chercheurs américains MH Dickson et MA Boettger qui nous ont fourni certaines de leurs lignées;

- P Touzard (établissements Clause) qui, ayant luimême entrepris une étude analogue, nous a communiqué ses meilleurs géniteurs;

- H Bannerot (INRA-Versailles) qui nous a conseillés, et aidés à tirer quelques lignées de ses croisements interspécifiques. Qu'il nous pardonne d'avoir maltraité sa variété Elsa qui, malgré sa germination délicate, reste la première variété moderne obtenue dans le type "flageolet vert" cher aux consommateurs français.

\section{RÉFÉRENCES}

Dickson MH (1972) Breeding beans for germination and growth at lower temperatures. Ann Rep Bean Improv Coop 15, 49

Dickson MH, Boettger MA (1979) Release of 12 root rot tolerant snap bean lines. Ann Rep Bean Improv Coop 22, 102

Ginoux JP (1981) Étude des relations hôte-parasite dans le couple Phaseolus vulgaris - Pythium ultimum. Thèse doctorat d'université, USTL Montpellier, $199 \mathrm{p}$

Hagedorn DJ, Rand RE (1978) Developing beans resistant to Wisconsin's root rot complex and bacterial brown spot. Ann Rep Bean Improv Coop 21, 59-61

Hardwick RC (1971) The emergence and early growth of french and runner beans (Phaseolus vulgaris and $P$ coccineus) sown on different dates. $J$ Hortic Sci 47, 395-410

Lebreton P, Jay M, Voirin B (1967) Sur l'analyse qualitative et quantitative des flavonoïdes. Chim Anal 49, 375-383

Messiaen CM, Barriere Y, Belliard Alonzo L, De la Tullaye B, Bouhot D (1977) Étude qualitative des Pythium dans quelques sols des environs de Versailles. Ann Phytopathol 9, 455-465

Prakken R (1977) Crosses with some Phaseolus varieties which are "constantly pattern color". Ann Rep Bean Improv Coop 20, 35-38

Schvester D, Rives M (1957) Résultats d'essais de traitements de semences de haricots contre la mouche des semis Hylemia cilicrura. Phytiat Phytopharm 6, 35-41 